\title{
Gender-Specific Beneficial Effects of Docosahexaenoic Acid Dietary Supplementation in G93A-SOD1 Amyotrophic Lateral Sclerosis Mice
}

\author{
Pascual Torres ${ }^{1} \cdot$ Daniel Cacabelos $^{1}$ - Jèssica Pairada ${ }^{1} \cdot$ Kylynda C Bauer $^{2,3} \cdot$ Jordi Boada $^{1} \cdot$ Laia Fontdevila $^{1}$. \\ Chiara Rossi $^{1} \cdot$ Monica Povedano $^{4} \cdot$ Isidre Ferrer ${ }^{5,6} \cdot$ Reinald Pamplona $^{1} \cdot$ B. Brett Finlay ${ }^{2,3,7} \cdot$ Manuel Portero-Otín $^{1}$ (D) \\ Victòria Ayala ${ }^{1}$
}

Published online: 21 November 2019

(C) The American Society for Experimental NeuroTherapeutics, Inc. 2019

\begin{abstract}
Docosahexaenoic acid (DHA) is an essential fatty acid modulating key nervous system functions, including neuroinflammation, and regulation of pre- and postsynaptic membrane formation. DHA concentration decreases in the lumbar spinal cord (LSC) of amyotrophic lateral sclerosis (ALS) patients and murine preclinical models. Using a dietary supplementation, we increased DHA levels ( $2 \%$ mean increase, $p<0.01)$ in the LSC of the familial ALS murine model B6SJL-Tg(SOD1*G93A) $1 \mathrm{Gur} / \mathrm{J}$. This DHAenriched diet significantly increases male mouse survival by $7 \%$ (average 10 days over 130 days of life expectancy), and delays motor dysfunction (based on stride length) and transgene-associated weight loss $(p<0.01)$. DHA supplementation led to an increased anti-inflammatory fatty acid profile $(c a 30 \%, p<0.01)$ and a lower concentration of circulating proinflammatory cytokine TNF- $\alpha(p<0.001$ in males). Furthermore, although DHA-treated mice did not exhibit generally decreased protein oxidative markers (glutamic and aminoadipic semialdehydes, carboxyethyllysine, carboxymethyllysine, and malondialdehydelysine), dietary intake of DHA reduced immunoreactivity towards DNA oxidative damage markers (8-oxo$\mathrm{dG}$ ) in the LSC. In vitro we demonstrate that DHA and $\alpha$-tocopherol addition to a model of motor neuron demise (neonatal rat organotypic spinal cord model under chronic excitotoxicity) also preserves motor neuron number, in comparison with untreated spinal cords. Also, beneficial effects on cell viability were evidenced for the motor neuron cell line NSC-34 in front of $\mathrm{H}_{2} \mathrm{O}_{2}$ insult $(p<0.001)$. Globally we show a sex-specific benefit of dietary DHA supplementation in the G93A ALS mouse model, compared with mice fed an isocaloric control or a n-3-depleted diet. These changes were associated with an increased DHA concentration in the LSC and were compatible with in vitro results showing DHA neuroprotective properties. These results suggest the need for further study on the interaction of gender-influenced biological parameters and DHA in ALS pathogenesis.
\end{abstract}

Key Words Polyunsaturated fatty acids $\cdot$ protein oxidative modification $\cdot$ neuroinflammation $\cdot$ estrogens $\cdot n-3$ fatty acids

Pascual Torres and Daniel Cacabelos are considered first-authors.

Manuel Portero-Otín

manuel.portero@mex.udl.cat

$\triangle$ Victòria Ayala

Victoria.Ayala@mex.udl.cat

1 Departament de Medicina Experimental, Institut de Recerca Biomèdica de Lleida-Universitat de Lleida, Av. Rovira Roure 80, 25198 Lleida, Spain

2 Department of Microbiology and Immunology, University of British Columbia, Vancouver, BC, Canada

3 Michael Smith Laboratories, University of British Columbia, Vancouver, BC, Canada
4 Functional Unit of Amyotrophic Lateral Sclerosis (UFELA), Service of Neurology, Bellvitge University Hospital, Hospitalet de Llobregat, Spain

5 Department of Pathology and Experimental Therapeutics, University of Barcelona, Hospitalet de Llobregat, Spain

6 Biomedical Network Research Center on Neurodegenerative Diseases (CIBERNED), Institute Carlos III, Hospitalet de Llobregat, Spain

Department of Biochemistry and Molecular Biology, University of British Columbia, Vancouver, BC, Canada 


\section{Introduction}

Characterized by a progressive loss of upper and/or lower motor neurons, amyotrophic lateral sclerosis (ALS) is the motor neuron disease with the highest incidence amongst adult humans. Neuronal loss leads to muscular atrophy, paralysis, and death after a median disease duration of 3 years. Although $90 \%$ of the cases are not associated to heritable mutations (sporadic ALS or sALS), the remaining 10\% are related to mutations and inherited directly (familial ALS or fALS) [1]. One of the earliest genes whose mutations were associated with fALS was $\mathrm{Cu}, \mathrm{Zn}$-superoxide dismutase [2]. Genetic findings led to the development of the G93A-hSOD1 transgenic mouse as an animal model of motor neuron disease [3]. Moreover, sex differences are observed in ALS, with higher incidence and prevalence in men than in women, and distinct clinical phenotypes (men exhibit faster progression) [4]. These findings are also present in experimental ALS models [5].

Docosahexaenoic acid (DHA) [22:6(n-3)] is an n-3 polyunsaturated fatty acid (n-3 PUFA). In humans, DHA precursors are essential fatty acids. DHA precursors must be ingested from dietary sources, as essential fatty acids cannot be synthesized de novo in human cells. DHA is usually localized in cell membranes as a phospholipid component. The central nervous system (CNS) is especially rich in DHA [6]. Here, DHA regulates inflammatory processes [7], regulates formation of pre- and postsynaptic membrane components and cerebral hemodynamics $[8,9]$, and also promotes developmental processes [10] and memory [11].

Although underlying causes of motor neuron degeneration in ALS remain largely unknown [4], DHA is depleted in spinal cord postmortem samples of ALS patients, suggesting a contribution of changes in fatty acid metabolism in the pathogenesis of ALS [12]. However, the contribution of DHA depletion to the disease remains largely uncharacterized. Alternatively, previous work on DHA metabolism and neurodegeneration demonstrates a positive role of this lipid in Parkinson's disease [13], neuroinflammation [14], cognitive impairment [15], Alzheimer's disease [16], and neuropathic pain [17]. Previously, we showed that a general change in polyunsaturated fatty acid content in diets could influence disease evolution in the B6SJL-Tg (SOD1-G93A)1Gur/J transgenic mice [18]. However, whether this was due to direct changes in DHA was unknown.

In this study, we evaluated the neuroprotective effect of dietary DHA supplementation in a preclinical model of motor neuron demise, the B6SJL-Tg (SOD1-G93A)1Gur/J transgenic mice [3]. We investigated whether DHA nutritional interventions modulated $n-3$ amounts in the spinal cord in the disease evolution, influencing survival, weight loss, motor function-related variables, and fatty acid profiles at 3 different ages (presymptomatic stage, disease onset, and endpoint).

\section{Methods}

\section{Animals and Diets}

A colony of the strain B6SJL-Tg (SOD1-G93A)1Gur/J (JAX catalog stock number 002726; from now on G93A) was purchased at The Jackson Laboratories (Bar Harbor, MN, USA). Mice were maintained in B6SJL backgrounds by male founder crossing with B6SJLF1/J. Genotyping was performed following the manufacturer's instructions. A total of 10 to 12 animals were used to assess survival analyses and 5 to 9 mice for analytical measurements. After genotyping and weaning (21 days), animals were placed at a 12:12-h dark/light cycle, at $22 \pm 2{ }^{\circ} \mathrm{C}$ temperature, and at $50 \% \pm 10$ relative humidity, in individual cages. Animals were weighed weekly. All diets were stored at $4{ }^{\circ} \mathrm{C}$ for its better preservation. All experimental diets were isocaloric and without nutritional deficits. After 9 days under control diet, mice were randomized to follow on the same control diet (\#PMI5015, PMI International LabDiet, St. Louis, MO), a low-n-3 diet (TD 00522, Harlan Teklad, Madison, WI), or the same low n-3 supplemented with $0.6 \%(\mathrm{w} / \mathrm{w})$ DHA (Martek Bioscience, Columbia, MD), as previously described [19, 20]. For food intake calculation, pellets were weighed and removed weekly. The intake of n-3 fatty acids was calculated by accounting individual food intake and the amount of linolenic acid plus DHA specified in the diet. Blood was obtained by tail vein puncture. For animal sacrifice, mice were anesthetized with $2.5 \%$ isoflurane. Spinal cords were rapidly excised, snap-frozen in liquid $\mathrm{N}_{2}$, and stored at $-80^{\circ} \mathrm{C}$. All experimental procedures were approved by the Institutional Animal Care Committee of the University of Lleida, according to local laws and to the Directive 2010/63/EU of the European Parliament. The minimal number of animals was calculated according to the deviation of fatty acid profiles in previous experiments [18].

\section{Genotyping}

Both DNA extraction and PCR-based genotyping have been previously described [18]. Briefly, tail DNA was extracted using the XNAT Kit (\# 2740, Sigma-Aldrich, St. Louis, MO). Primers employed for hSOD1 transgene genotyping were as follows: Il2F (IMR042) 5'-CTAGGCCACAGAAT TGAAAGATCT-3', IlR2 (IMR043) 5'-GTAG GTGGAAATTCTAGCATCATCC-3', hSODlF (IMR113) 5'-CATCAGCCCTAATCCATCTGA-3', hSODIR (IMR114) 5'-CGCGACTAACAATCAAAGTGA-3'. Murine IL2 genotyping was assessed as a technical positive control, as indicated by providers (JAX catalog stock number 002726). Thermal Cycler v2.08 (Applied Biosystems, Carlsbad, CA) was used to perform the following PCR protocol: predenaturation for $5 \mathrm{~min}$ at $95^{\circ} \mathrm{C}$, denaturation for $1 \mathrm{~min}$ at $94{ }^{\circ} \mathrm{C}$, annealing for $45 \mathrm{~s}$ at $50{ }^{\circ} \mathrm{C}$, elongation for $4 \mathrm{~min}$ at 
$72{ }^{\circ} \mathrm{C}$, and finally, after 35 cycles, elongation for another 7 min at $72{ }^{\circ} \mathrm{C}$. The amplification products were resolved in $1 \%$ agarose stained with SYBR safe (S33102, Thermo Fisher Scientific, Waltham, MA) following the manufacturer protocol and electrophoresed for 30 min under constant $100 \mathrm{~V}$. Images were taken under a 365-nm UV light lamp from Alpha Innotech (Santa Clara, CA) with the software acquisition Digidoc RT2 (Alpha Innotech, Santa Clara, CA).

\section{Stride Length Analysis}

Phenotypic analyses for motor neuron loss have been previously described [18]. Briefly, mice were trained at a flat narrow corridor ( $5 \mathrm{~cm}$ wide, $70 \mathrm{~cm}$ long) 3 times per week for 1 month. After this training, animal hind limbs were stained with a nontoxic colorant and put on a paper which served to track the walking behavior along the corridor. A minimum of 5 to 7 continuous strides was recorded to calculate the mean value of stride measurements for each animal 3 days per week. The first footprints were discarded as the mice tend to walk faster when they are released to the corridor. Stride and stance lengths were measured manually and in a blinded manner. Corridors were periodically ventilated to avoid crosscontamination of volatile compounds present in mice.

\section{Fatty Acid Compositional Analyses}

After controlled thawing, samples were kept at $4{ }^{\circ} \mathrm{C}$ on ice during the homogenization. A buffer containing $180 \mathrm{mM} \mathrm{KCl}, 5 \mathrm{mM}$ MOPS, $2 \mathrm{mM}$ EDTA, $1 \mathrm{mM}$ diethylenetriaminepentaacetic acid, and $1 \mu \mathrm{M}$ of freshly prepared butylated hydroxyl toluene (BHT) at $\mathrm{pH} 7.3$ was used, employing a 1:20 (sample weight:homogenization buffer volume ratio). A homogenizer device (T10 basic UltraTurraX, IKA, Staufen, Germany) was employed for mechanical homogenization (15 s at 15,000 rpm). Protein concentrations were measured by the Bradford method [21].

After the homogenization, lipids from the sample were extracted with chloroform/methanol $(2: 1, \mathrm{v} / \mathrm{v}) 0.01 \%$ BHT for 3 times as described in [22]. The chloroform phase was evaporated under $\mathrm{N}_{2}$. Then, the fatty acids were transesterified by incubation of lipids in freshly prepared $5 \%$ methanolic $\mathrm{HCl}$ for $90 \mathrm{~min}$ at $75^{\circ} \mathrm{C}$. The extraction of the resulting fatty acid methyl esters was performed by adding n-pentane and saturated $\mathrm{NaCl}$ solution $(2: 1 \mathrm{v} / \mathrm{v})$. The n-pentane phase was removed under nitrogen. The remaining product was dissolved in $80 \mu \mathrm{l}$ of $\mathrm{CS}_{2}$ for gas chromatography. Separation was assessed by a DBWAX capillary column $(30 \mathrm{~m} \times 0.25 \mathrm{~mm} \times 0.20 \mu \mathrm{m})$ in an Agilent GC System 7890A with a Series Injector 7683B and a flame ionization detector (Agilent Technologies, Santa Clara, CA). The injection port was maintained at $220{ }^{\circ} \mathrm{C}$ and the detector at $250{ }^{\circ} \mathrm{C}$. The temperature program was as follows: 2 min at $100^{\circ} \mathrm{C}$, then an increase of $10^{\circ} \mathrm{C} / \min$ to $200^{\circ} \mathrm{C}$, then
$5{ }^{\circ} \mathrm{C} / \mathrm{min}$ to $240{ }^{\circ} \mathrm{C}$, and finally maintained at $240{ }^{\circ} \mathrm{C}$ for $10 \mathrm{~min}$. For fatty acid methyl ester integration, we compared the peaks with the retention time of authentic standards (Larodan Fine Chemicals, Malmö, Sweden) injected according to the same method. Results were expressed as mol\%.

The fatty acid-derived indexes [22] were calculated with the following formulas: Saturated fatty acids (SFA) $=\Sigma \%$ of saturated fatty acids; unsaturated fatty acids (UFA) $=\Sigma \%$ unsaturated fatty acids; monounsaturated fatty acids $($ MUFA $)=\Sigma \%$ of monoenoic fatty acids; polyunsaturated fatty acids series n-3 (PUFAn-3) $=\Sigma \%$ of polyunsaturated fatty acids n-3 series; polyunsaturated fatty acids series n-6 (PUFAn-6) $=\Sigma \%$ of polyunsaturated fatty acids $n-6$ series; average chain length $(\mathrm{ACL})=[(\Sigma \%$ total $14 \times 14)+\cdots+(\Sigma$ $\%$ total $n \times n)] / 100(n=$ carbon atom number $)$; peroxidizability index $(\mathrm{PI})=[(\Sigma$ mol\% monoenoic $\times$ $0.025)+(\Sigma$ mol\% dienoic $\times 1)+(\Sigma$ mol\% trienoic $\times 2)+(\Sigma$ mol\% tetraenoic $\times 4)+(\Sigma$ mol\% pentaenoic $\times 6)+(\Sigma$ mol\% hexaenoic $\times 8)]$; double-bond index $(\mathrm{DBI})=[(\Sigma \mathrm{mol} \%$ monoenoic $)+(2 \times \Sigma$ mol\% dienoic $)+(3 \times \Sigma$ mol\% trienoic $)+(4 \times \Sigma \%$ mol tetraenoic $)+(5 \times \Sigma$ mol\% pentaenoic $)+(6 \times \Sigma$ mol\% hexaenoic $)$; anti-inflammatory in$\operatorname{dex}(\mathrm{AI})=(20: 3 n-6)+(20: 5 n-3)+(22: 5 n-3)+(22: 6 n-3) /$ (20:4n-6). This index accounts for potential changes in cell membrane phospholipids (altering the substrate of cyclooxygenases from arachidonic acid to EPA/DHA), providing eicosanoids and other derivates that have lower inflammatory potential, as specific prostaglandins (PGE3) and other products [23].

\section{Protein Oxidative Modifications}

The concentration of protein oxidative modifications glutamic semialdehyde (GSA), aminoadipic semialdehyde (AASA), glycoxidative modification carboxyethyllysine (CEL), mixed lipooxidative glycoxidative modification carboxymethyllysine (CML), and lipooxidative modification malondialdehyde lysine (MDAL) was analyzed by gas chromatography-mass spectrometry (GC/MS) in lumbar spinal cord homogenates as indicated [22]. Briefly, $0.5 \mathrm{mg}$ of proteins from lysate was reduced with an overnight incubation in $500 \mathrm{mM} \mathrm{NaBH}_{4}$ (final concentration) in $0.2 \mathrm{M}$ borate buffer, containing a 1 drop of hexanol as an antifoam reagent. Reduced proteins were then re-precipitated with $20 \%$ trichloroacetic acid (final concentration). Internal standards used for relative quantification were the following: $\left[{ }^{2} \mathrm{H}_{8}\right]$ lysine $\left(d_{8^{-}}\right.$ Lys; CDN isotopes); $\left[{ }^{2} \mathrm{H}_{4}\right] \mathrm{CML}\left(d_{4}-\mathrm{CML}\right),\left[{ }^{2} \mathrm{H}_{4}\right] \mathrm{CEL}\left(d_{4}\right.$ CEL), $\left[{ }^{2} \mathrm{H}_{8}\right]$ MDAL $\left(d_{8}\right.$-MDAL), $\left[{ }^{2} \mathrm{H}_{5}\right] 5$-hydroxy-2aminovaleric acid (for GSA quantitation), and $\left[{ }^{2} \mathrm{H}_{4}\right] 6$-hydroxy-2-aminocaproic acid (for AASA quantitation). Proteins were hydrolyzed by incubation at $155^{\circ} \mathrm{C}$ for $30 \mathrm{~min}$ in $1 \mathrm{ml}$ of $6 \mathrm{~N} \mathrm{HCl}$. Samples were dried using a Speed-Vac centrifugal evaporator (SPD131DDA; Thermo 
Fisher Scientific, Waltham, MA). For the preparation of the methyl esters, the hydrolysates were dissolved in $1.5 \mathrm{ml}$ of freshly prepared $1 \mathrm{~N}$ methanolic $\mathrm{HCI}$ and heated for $30 \mathrm{~min}$ at $65{ }^{\circ} \mathrm{C}$. The solvent was evaporated at room temperature under a stream of $\mathrm{N}_{2}$; then, $1.5 \mathrm{ml}$ trifluoroacetic anhydride was added, and the mixture was incubated at room temperature for $1 \mathrm{~h}$. The resulting N, O-trifluoroacetyl methyl ester derivatives were analyzed in Agilent model 6890 gas chromatograph equipped with a 30-m HP-5MS capillary column $(30 \mathrm{~m} \times 0.25 \mathrm{~mm} \times 0.25 \mu \mathrm{m})$ coupled to an Agilent model 5973A mass selective detector (Agilent, Barcelona, Spain) under the conditions previously described [18].

\section{Analysis of Cytokines in Plasma}

After blood obtention, it was centrifuged at $8000 \mathrm{rpm}$ for 6 min to retrieve plasma. Plasma was stored at $\leq-20{ }^{\circ} \mathrm{C}$ until cytokine analysis. Cytokine levels from plasma were measured using the BD Biosciences Cytometric Bead Array Mouse Inflammation Kit (BD, Franklin Lakes, NJ, USA).

\section{Organotypic Spinal Cord Culture}

Lumbar spinal cord slices from P8 rat pups were obtained as described [24]. Slices were maintained in 50\% minimal essential medium, 25 mM HEPES, 25\% Hank's balanced salt solution with D-glucose $25.6 \mathrm{mg} / \mathrm{l}, 25 \%$ heat-inactivated horse serum, and $2 \mathrm{mM}$ L-glutamine (Invitrogen, Carlsbad, CA, USA). Five complete slices from the lumbar region were transferred to 30-mm-diameter Millipore Millicell-CM $(0.22 \mu \mathrm{m}$; Millipore Corporation, Bedford, MA, USA) membrane inserts. The inserts were placed in 6-well culture trays (35-mm-diameter Falcon; BD), with 1-ml culture medium: $50 \%$ (vol/vol) minimal essential medium (MEM) with $25 \mathrm{mM}$ HEPES (Invitrogen), 25\% (vol/vol) heat-inactivated horse serum, and 25\% (vol/vol) Hank's balanced salt solution (HBSS) supplemented with D-glucose $(25.6 \mathrm{mg} / \mathrm{ml}$; Sigma, St. Louis, MO, USA), $2 \mathrm{mM}$ L-glutamine, $100 \mathrm{U} / \mathrm{ml}$ penicillin, and $100 \mu \mathrm{g} / \mathrm{ml}$ streptomycin (Invitrogen). Organotypic cultures were incubated at $37{ }^{\circ} \mathrm{C}$ in a $5 \% \mathrm{CO}_{2}-95 \% \mathrm{O}_{2}$ humidified incubator, and the culture medium was changed $4 \mathrm{~h}$ after harvesting and then twice a week. Cultures were let to stabilize for 10 days; after this point, the motoneuron population reaches a steady number and remains stable from 1 to 4 weeks [24]. Thus, the treatments, inducing chronic excitotoxicity with threohydroxyaspartate (THA) at $100 \mu \mathrm{M}$, with DHA and/or $\alpha$-tocopherol, $500 \mu \mathrm{M}$ each, started 10 days after the explant procedure. After 15 days of treatment, cultures were harvested and fixed in $4 \%$ paraformaldehyde in $0.1 \mathrm{M}$ phosphate buffer, $\mathrm{pH} 7.4$, overnight at $4{ }^{\circ} \mathrm{C}$ and processed for immunohistochemistry.

\section{NSC-34 Cell Culture and Viability}

NSC-34 (kindly given by Prof. JE Esquerda, University of Lleida, Spain) were grown in MEM-Advanced medium (Invitrogen) supplemented with $10 \%$ heat-inactivated fetal bovine serum (Invitrogen), $2 \mathrm{mM}$ L-glutamine (Invitrogen), and $20 \mathrm{U} / \mathrm{ml}$ penicillin and $20 \mu \mathrm{g} / \mathrm{ml}$ streptomycin (Invitrogen) as antibiotics. Cells were kept at $37{ }^{\circ} \mathrm{C}$ in humidified atmosphere with $5 \%$ of $\mathrm{CO}_{2}$ [24]. Cell viability in cell cultures was determined by using the MTT-based Cell Toxicity Colorimetric Assay Kit (Sigma) according to the manufacturer's instructions. The results were expressed as the percentage of viability versus untreated cells.

\section{Immunohistochemistry}

Animals were anesthetized by an intraperitoneal injection of pentobarbital and ketamine, $20 \mathrm{mg} / \mathrm{kg}$ and $60 \mathrm{mg} / \mathrm{kg}$ respectively in PBS. Mice were then perfused with saline solution followed by ice-cold 4\% paraformaldehyde (Sigma-Aldrich, St. Louis, MO, USA) solution (freshly prepared with $\mathrm{pH} 7.4$ phosphate buffer). Spinal cords were extracted and fixation was continued by incubation in $4 \%$ paraformaldehyde made in $\mathrm{pH} 7.4$ phosphate buffer overnight at $4{ }^{\circ} \mathrm{C}$. The next day, samples were cryopreserved in $30 \%$ sucrose (made in $\mathrm{pH} 7.4$ phosphate buffer) for $48 \mathrm{~h}$. After this time, tissue was encased in a cubic recipient (Peel-A-Way Disposable Embedding Molds-S-22, Polysciences Inc., Warrington, PA), and embedded in tissue-freezing medium (Triangle Biomedical Sciences Inc., Newcastle, UK) and frozen $\left(-80^{\circ} \mathrm{C}\right)$. The lumbar spinal cord was then cut at a $16-\mu \mathrm{m}$ section depth and resulting seeded on a gelatin-coated slide. In the case of organotypic slices, these were directly seeded onto the slides. Samples were permeabilized with $0.4 \%$ Triton X-100 PBS for $30 \mathrm{~min}$ and blocked with $5 \%$ normal horse serum in $0.4 \%$ Triton $\mathrm{X}$ 100 PBS (blocking solution) for $2 \mathrm{~h}$ at room temperature. Primary antibody (diluted according to manufacturer's instructions) incubation, anti-8-oxo-dG (ab62623, Abcam, Cambridge, UK), anti- $\gamma \mathrm{H} 2 \mathrm{Ax}$ (ab2893, Abcam), anti-GFAP (ab7260, Abcam) anti-Ibal (ab5076, Abcam), and anti-SMI32 (Covance, Princeton, NJ), was performed in blocking solution overnight at $4{ }^{\circ} \mathrm{C}$. Then, the slices were washed with PBS $3 \times$ for $10 \mathrm{~min}$ at room temperature, followed by the secondary antibody (diluted 1:800 in PBS), goat anti-mouse Alexa Fluor 555 (A21422, Thermo Fisher Scientific, Waltham, MA) and/or goat anti-rabbit Alexa Fluor 488 (A11008, Thermo Fisher Scientific, Waltham, MA), incubation for $1 \mathrm{~h}$ at room temperature in darkness. Sections were finally counterstained with 4,6-diamidino-2-phenylindole dihydrochloride (DAPI, $1 \mu \mathrm{g} / \mathrm{ml}$ ) in PBS for $5 \mathrm{~min}$ at RT and mounted on slides with Vectashield (Vector Laboratories, Burlingame, CA). In selected sections, the 
primary antibody was omitted to assure labeling specificity. Confocal microscopy was performed with a Fluoview 1000 microscope (Olympus Corporation, Tokyo, Japan) for immunofluorescence imaging. Immunoreactivity quantification and motor neuron count (in the case of organotypic culture) were analyzed with the ImageJ software [25], by selecting motor region from anterior horns in the spinal cord. Motor neurons were putatively identified by soma size, low DAPI fluorescence, and anterior location. All measurements and analyses were done in a double-blinded fashion.

\section{Statistical Analyses}

All data are plotted on graphs as mean \pm S.E.M. All statistics were performed using the SPSS software (SPSS Inc., Chicago, IL) or the Prism software (GraphPad Software, San Diego, CA). Differences between groups were analyzed by the Kaplan-Meyer analyses, Student's $t$ tests, or ANOVA with post hoc analyses, after normality assessment by the Kolmogorov-Smirnoff test. Correlations between variables were evaluated by Pearson's statistic. The 0.05 level was selected as the point of minimal statistical significance in every comparison.

\section{Results}

\section{Dietary DHA Supplementation Extends G93A Mouse Survival, Prevents Weight Loss, and Improves Motor Function in a Sex-Specific Manner}

In order to determine the potential benefits of DHA supplementation, we analyzed the survival of ALS mice by sex and diet. The Kaplan-Meyer analysis revealed a longer survival of G93A male mice survival under DHA-supplemented diet (Fig. 1a). The low-n-3 diet did not induce a significant loss in survival (Fig. 1a). Female G93A mice survival did not exhibit any differences between the 3 diet groups analyzed (Fig. 1a). Moreover, weight loss, indicating motor neuron impairment in this model, was significantly influenced by diet in G93A male mice (Fig. 1b), but not in females (Fig. 1b). Regarding the motor function, we also assessed gait strides to determine the impact of diet on motility. Results indicate a slower decrement on stride length and stance length in G93A male mice under DHA-supplemented diet compared with those under control and low-n-3 diets (Fig. 1c and d) and a lower effect of diet and motor function interaction in female G93A mice (Fig. $1 \mathrm{c}$ and d). Noteworthy, food intake, revealing motor function, also showed gender-influenced differences to diets (Fig. 1e).

\section{DHA Dietary Supplementation Increases DHA Contents and Decreases AA Concentrations in the LSC of G93A Mice}

To confirm DHA bioavailability in the LSC and the feasibility to modify fatty acid content using dietary intervention, we measured LSC fatty acid profile. A significant correlation was present between the n-3 intake (DHA + linolenic) and LSC DHA content, irrespective of gender (Fig. 2a) before disease onset (age < 90 days). We then compared LSC fatty acid composition in the presymptomatic stage (60 days), at disease onset (90 days) and endpoint. Fatty acid profile analysis of LSC revealed that DHA content in LSC decreases according to disease advancement. In contrast, DHA supplementation induces a significant increase of DHA concentration in LSC at 60 days and 90 days and a decrease of this fatty acid under the low-n-3 diet (Fig. 2b). Moreover, in males, DHA supplementation increased DHA content in LSC at all times, an effect not exhibited in females (Fig. 2b). In contrast with the increase of DHA, arachidonic acid (AA) content showed a statistically significant decrease in the group with the DHA-supplemented diet either at a presymptomatic stage, at disease onset, and at endpoint both in males and in females (Fig. 2c).

\section{Diet Influences Protein Oxidative Damage}

Previous data show that oxidative modifications are sensitive to changes in dietary fatty acid profile [18]. In order to evaluate protein oxidative modifications of LSC, we quantified by GC-MS the concentrations of GSA, AASA, CML, CEL, and MDAL, representative markers of protein modifications. GSA was higher in 60-day-old mice, but reduced at 90 days and endpoint in LSC from G93A mice under DHA diet mice, compared with low-n-3 diet both in males and females (Fig. 3a). AASA is decreased at 60-day-old mice in the DHA diet group in males and females (Fig. 3b). AASA showed an increase at 90 days and endpoint in the DHA diet group compared with a presymptomatic stage in transgenic males and females, but AASA decrease at endpoint was only observed in female DHA-treated mice. CML quantification (Fig. 3c) in G93A mice fed with DHA diet resulted in reduced levels at 60 days old, respective to the male mice fed a control diet group in male or female mice on the low-n-3 diet. At 90 days old, G93A male mice fed with DHA diet showed reduced levels of CML compared with those fed with low-n3 diet. G93A females exhibited a reduction compared with the control diet group but were not different in the low-n- 3 group. At endpoint, diet did not influence in CML content of transgenic male mice. In contrast, CML levels in transgenic female mice were lower in the DHA diet group. Interestingly, age was not a significant factor influencing CML content. A decrease in CEL levels was only observed in female mice at the 60-day 


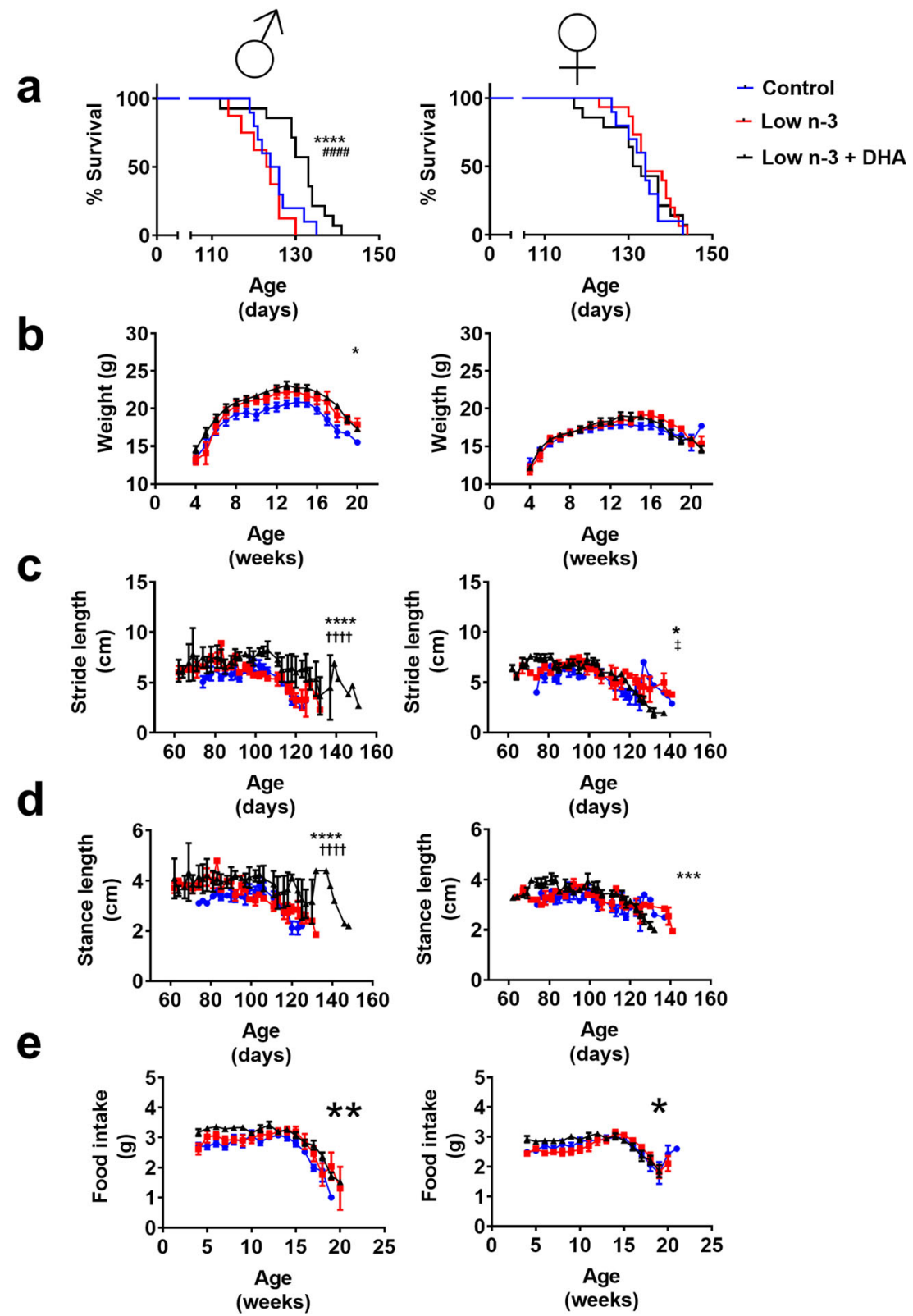

Fig. 1 DHA dietary supplementation increases the survival of G93A male mice. DHA supplementation increases percent survival in males (a) but not in females. Growth curves also show diet-induced differences in males (b), though age at the maximum weight is not altered. Disease evolution, as measured by loss of stride (c) and stance (d) lengths, and daily food intake (e), also indicates the protective effect of dietary DHA supplementation in males, but not in females. Percent survival in (a) was estimated by Kaplan-Meyer analyses. Two-way ANOVA with repeated measures was employed for $(\mathbf{b}),(\mathbf{c}),(\mathbf{d})$, and (e). In the case of $(\mathbf{b})$, in males, this analyses showed a $p=0.0119$ for diet effect and $p<0.0001$ for age effect, and diet and age interaction is not significant; for females, $p=$ 0.6278 for diet effect, $p<0.0001$ for age effect; but in this case, age and diet interaction being significant, $p=0.0046$. For (c), in males, diet effect showed a $p=0.031$, age effect $p=0.0007$, and age and diet interaction

was not significant; for females, diet effect showed a $p=0.22$, with age effect $p<0.0001$, and age and diet interaction with $p=0.037$. For $(\mathbf{d})$, in males, age effect was $p=0.01$ with diet $p=0.13$ and interaction between age and diet $p=0.21$; for females, age effect was $p=0.0011$, diet factor $p=0.15$, and interaction between age and $\operatorname{diet} p=0.027$. For (e), in males, this analyses showed a $p=0.0011$ for diet effect, $p<0.0001$ for age effect, and $p=0.048$ for diet and age interaction; for females, a $p=0.1395$ for diet effect, $p<0.0001$ for age effect, and age and diet interaction being significant, $p<0.0001$. In all cases, matching between individuals was effective $(p<0.0001)$. Error bars represent \pm S.E.M. $* * * * p<0.0001$, $* * * p<0.001$, and $* p<0.05$ in control versus low- $n-3+D H A$ diet comparisons, whereas ${ }^{\dagger \dagger} p<0.0001$ in low- $n-3$ versus low-n-3 + DHA diet comparisons and ${ }^{\ddagger} p<0.05$ in control versus low-n-3 diet groups in post hoc multiple comparisons

time point (Fig. 3d). At 90 days, both males and females of the DHA group exhibited lower CEL levels than low-n-3 diet

group, with CEL levels comparable between the control and DHA-supplemented mice. At endpoint, males under DHA 
Fig. 2 Dietary DHA

supplementation increases DHA

contents and lowers AA

concentrations in the lumbar

spinal cords of G93A mice. (a)

DHA content of the lumbar spinal cords correlates significantly (see inside the graph for Pearson correlation coefficients) with dietary intake of $n-3$ fatty acids in 90-day-old males (linear regression: $\left.p<0.001 ; r^{2}=0.58\right)$ and females (linear regression: $\left.p<0.001 ; r^{2}=0.24\right)$.

Consequently, across different ages, males supplemented with DHA have higher DHA levels in LSC with similar results in females, with the exception of endpoint period (b). Similarly, AA is reduced in LSC in male mice under the DHA diet, as well as in females (c). Bars indicate mean values, whereas error bars represent S.E.M. Significant differences between dietary groups $(* p<0.05, * * p<0.01$, $* * * p<0.001)$ were evaluated by a Bonferroni post hoc analysis after 2-way ANOVA, accounting age and diet a
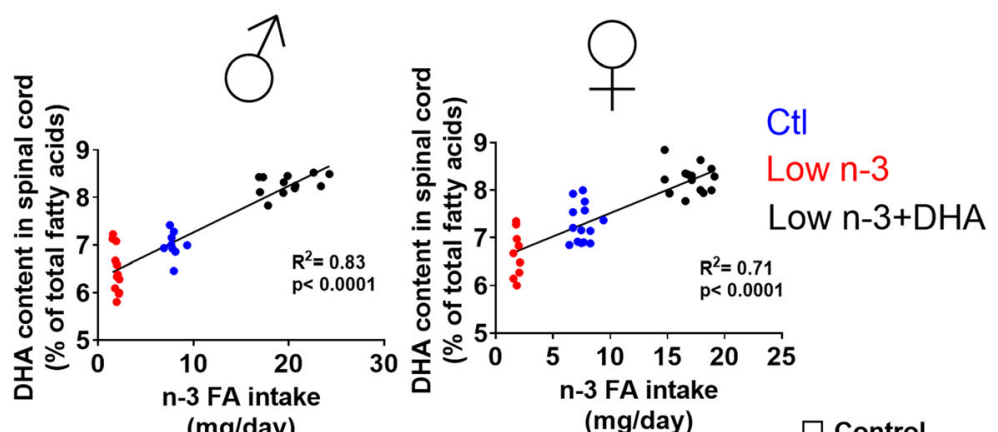

b
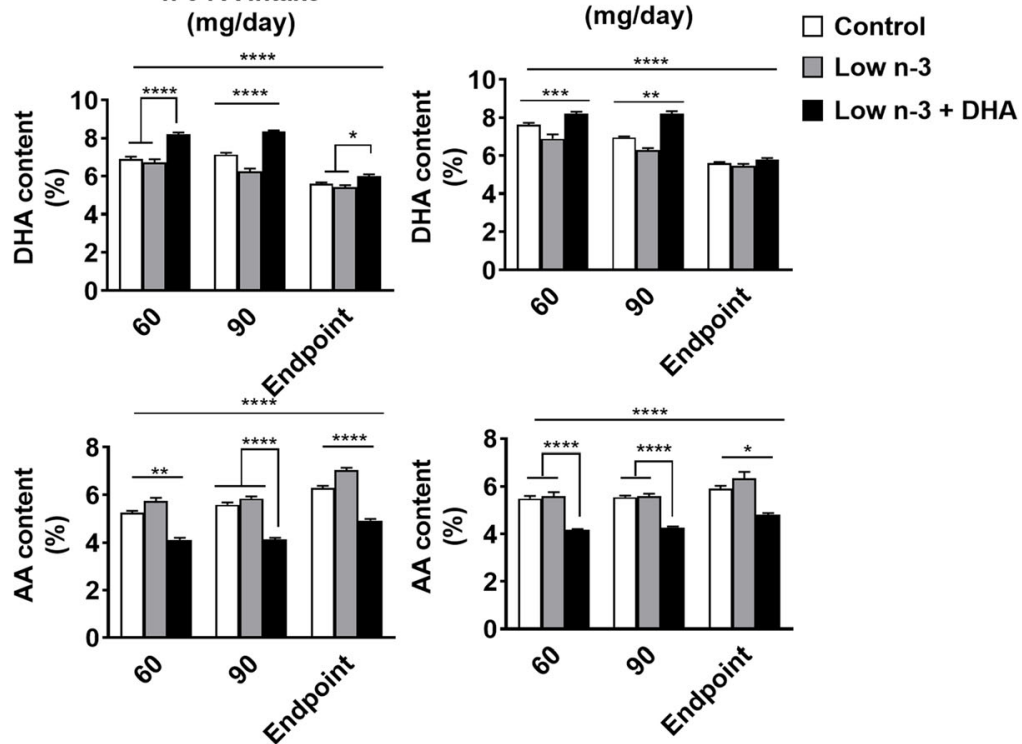

supplementation did not show significant differences in CEL content compared with the other diet groups. Nevertheless, females fed with DHA diet exhibited lower levels of CEL compared with those fed with the low-n-3 diet, but not the control diet group. In this case, age was a relevant factor for CEL levels in LSC of males and females. Finally, G93A male mice fed with DHA diet exhibited reduced levels of MDAL (Fig. 3e) at the presymptomatic stage compared with those fed with control and low-n-3 diets; this reduction was not present in females. At 90 days old, diet did not induce any changes in MDAL content in males. However, females under the DHA diet exhibited lower levels of this marker than those fed with the control diet. At endpoint, there were no significant differences of MDAL levels for males in all experimental groups. DHA supplementation diminished MDAL levels in female mice at all time points. These results suggest that MDAL modulates ALS disease progression in G93A female, but not in male, mice.

\section{DHA-Enriched Diet Influences Inflammatory Constraints in LSC and Plasma}

To explore other mechanisms explaining DHA beneficial effects, we evaluated the potential inflammatory modulation properties of DHA intake. Thus, anti-inflammatory index of LSC fatty acid profiles was increased on the DHA-enriched diet groups (Fig. 4a). Interestingly, this index was decreased at the endpoint stage in all groups. Of note, and reinforcing the complexity of gender-diet interaction, anti-inflammatory index did not show gender differences; it was equally increased in males and females.

To focus on the potential mechanisms leading to DHA survival effects, further experiments were performed in male mice. In order to confirm the role of DHA intake as an inflammation modulator, we quantified plasma concentrations of IL12 , TNF- $\alpha$, IFN- $\gamma$, MCP-1, IL-10, and IL- 6 at 90 days. These analyses showed a statistically significant influence of DHA dietary enrichment in plasma TNF- $\alpha$ concentrations (Fig. 4b).

\section{Effect of Dietary DHA Could Involve Several Mechanisms}

DHA exerts pleiotropic functions, including interaction with synaptic components [26]. To evaluate this potential function, we evaluated syntaxin 3 levels in male G93A mice. DHA supplementation enhanced syntaxin 3 concentrations in LSC at early stages of motor neuron dysfunction (Fig. 5a). The concentration of other neuron-specific components, such as 
Fig. 3 Heterogenic effects of DHA dietary supplementation over protein oxidative modifications in the proteins of LSC of G93A mice. Oxidative damage as measured by GSA (a) and AASA (b) contents in LSC proteins shows gender- and agesensitive profiles. In contrast, CML, a marker of mixed lipooxidative and glycoxidative modifications (c), and CEL, a marker of methylglyoxal-derived modifications (d), generally exhibit decreased levels at 90-day and endpoint stages, irrespective of gender. Noteworthy, MDAL, a marker of lipooxidative modifications, shows a significant decrease in males only at earlier stages (e). Bars indicate mean values, whereas error bars represent \pm S.E.M. Significant differences between dietary groups $(* p<0.05, * * p<0.01$, $* * * p<0.001, p<0.0001)$ were evaluated by a Bonferroni post hoc analysis after 2-way ANOVA, accounting age and diet
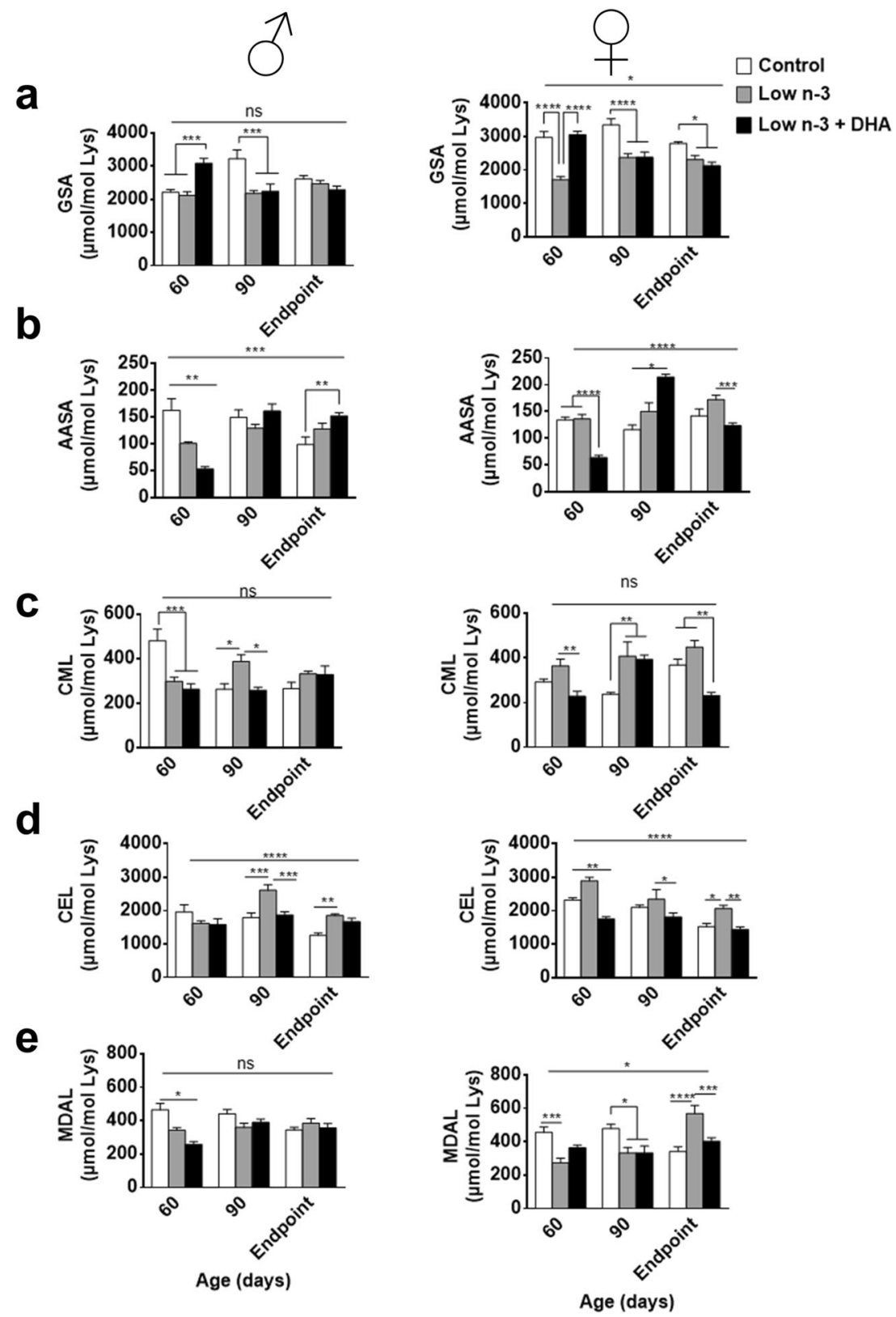

neurofilaments (reacting to SMI-32 antibody), was also enhanced by DHA treatment, as shown by confocal microscopy imaging (Fig. 5b). This was associated with increased ubiquitin immunoreactivity, suggesting enhanced protein repair (Fig. 5 b). Indeed even DNA show increased oxidative damage in G93A mice [27]. We evaluated the presence of 8-oxo-7,8dihydro-2'-deoxyguanosine (8-oxo-dG), a direct marker of oxidative modification of DNA in LSC, focused to 90-day male group comparing control diet and DHA-supplemented diet. 8-Oxo-dG immunoreactivity was significantly lower in spinal cord slices from animals fed with DHA diet compared with those fed with the control diet (Fig. 5c). The DNA damage response is a mechanism to combat threats posed by DNA damage, induced in ALS [28]. To evaluate whether this mechanism was affected by dietary DHA intake, we utilized confocal microscopy to assess the amount of $\gamma \mathrm{H} 2 \mathrm{Ax}$ foci, a surrogate for DNA damage response. $\gamma \mathrm{H} 2 \mathrm{Ax}$ immunoreactivity in LSC of 90-day male ALS mice was lower in 90-day-old G93A male mice fed with the DHA diet when compared with those mice fed with the control diet (Fig. 5c).

In order to evaluate further mechanisms of DHA effects, we evaluated GFAP immunostaining, an indication of astrogliosis, related to disease activity [29] . The results show that, at 90 days, DHA supplementation did not induce a significant change in the levels of glial proliferation (Fig. 5d). Similarly, we evaluated the percentage of anti-Iba1-positive 
Fig. 4 DHA diet influences inflammatory constraints in G93A mice. Anti-inflammatory indexes of fatty acid profiles from LSC of G93A mice are significantly enhanced by DHA supplementation, irrespective of age and gender (a). In line with this, plasmatic TNF- $\alpha$ concentration is also decreased in 90-day-old G93A male mice (b). Bars indicate mean values, whereas error bars represent \pm S.E.M. In (a), significant differences between dietary groups $(* p<0.05$, $* * * p<0.001, * * * * p<0.0001)$ were evaluated by a Bonferroni post hoc analysis after 2-way ANOVA, accounting age and diet. In (b), differences between dietary groups were evaluated by an ANOVA analysis followed by a Bonferroni post hoc analysis, being $* * * p<0.001$
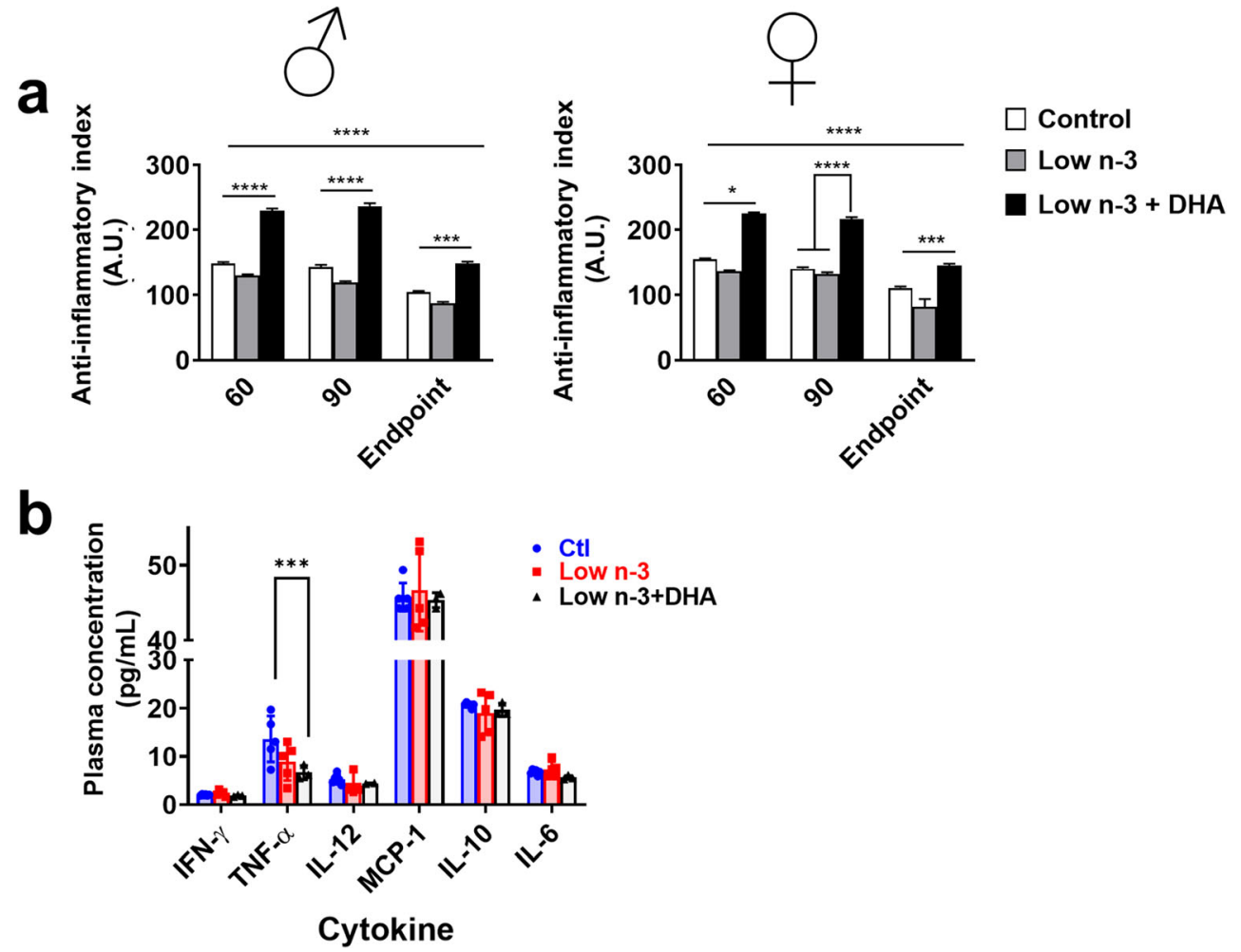

cells, as a marker for microglia infiltration [30]. These analyses show that low n-3 intake, with our without DHA addition, diminishes the percentage of anti-Iba1-positive cells (Fig. 5e).

\section{Neuroprotective DHA Effects Are Extended to Other Motor Neuron Disease Models}

In order to evaluate if DHA protective effects could be extended to other models of motor neuron demise, we evaluated the effect of DHA supplementation in 2 different cellular models of motor neuron death: in chronic excitotoxicity in vitro and in the motor neuron-like cell line NSC-34. The results show that, in organotypic cultures under chronic excitotoxicity impinged by THA treatment, DHA alone was not able to reverse motor neuron loss, but in the presence of $\alpha$-tocopherol (Fig. 6a). However, DHA alone was able to prevent the toxicity of $\mathrm{H}_{2} \mathrm{O}_{2}$ in NSC-34 in a significant manner (Fig. 6b).

\section{Discussion}

We show a gender-diet interaction in the G93A-hSOD1 mouse model of ALS. Dietary DHA supplementation has beneficial effects in male survival, slowing ALS progression and improving motor function.

Previous data suggests the influence of gender in dietary PUFA changes in ALS progression, reinforcing the importance of the diet-gender interaction in ALS [18]. Further, there are marked differences in neuroinflammatory constraints between genders in mice [31]. Thus, estrogen-sensitive differences in microglia and lymphocyte biology may explain differences in neurodegeneration. Studies also indicate estrogendependent pathways enhancing DHA production in females versus males [32]. In light of these previous evidence, our data suggest that in G93A mice, dietary DHA is able to modulate sex-associated neuroinflammation constraints. Globally, DHA supplementation improves some neurodegenerative disorders [19], providing an effective therapeutic treatment in a model of spinal cord injury [33]. We also observed that DHA supplementation mitigates oxidative stress markers in the ALS spinal cord. Although the evidence of beneficial effects of DHA in the CNS exists, diets increased in eicosapentaenoic acid (EPA)- a precursor of DHA- decreased survival in G93A mice [34]. Therefore, the present study highlights the relevance of specific effects of the different n-3 fatty acids in diet regarding central nervous system metabolism. In contrast to DHA supplementation, EPA exposure is not able to decrease arachidonic acid content in the spinal cord [34]. Nevertheless, AA is required for proinflammatory signaling and significantly decreasing LSC AA levels could potentially prevent an exacerbation of cyclooxygenase-mediated inflammation [35]. Moreover, earlier findings report that DHA-enriched diet induces beneficial changes on mitochondrial metabolism that EPA diet cannot reproduce [36]. Our experiments prove the feasibility of increasing DHA content in LSC of G93A mice within a dietary supplementation. In contrast to other 
a

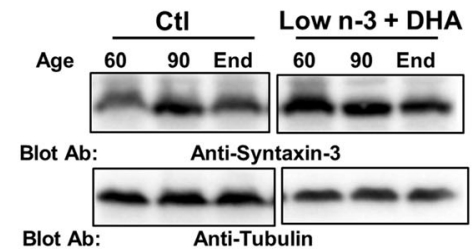

b

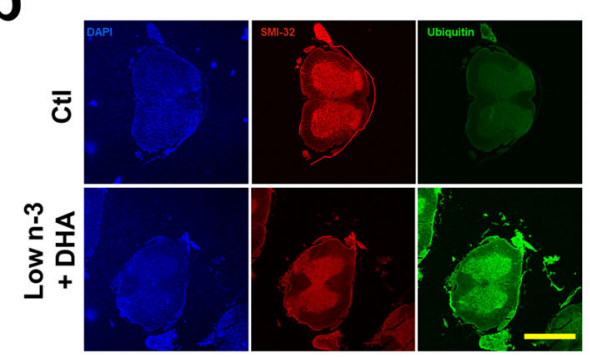

C

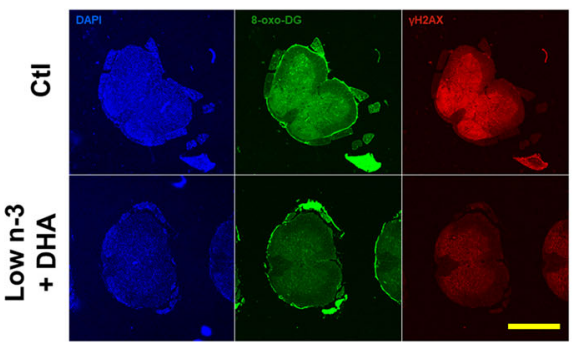

d

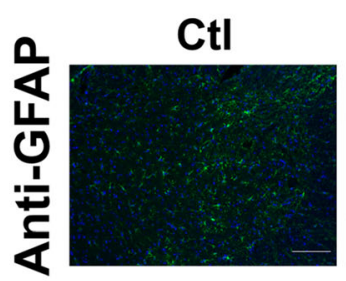

e
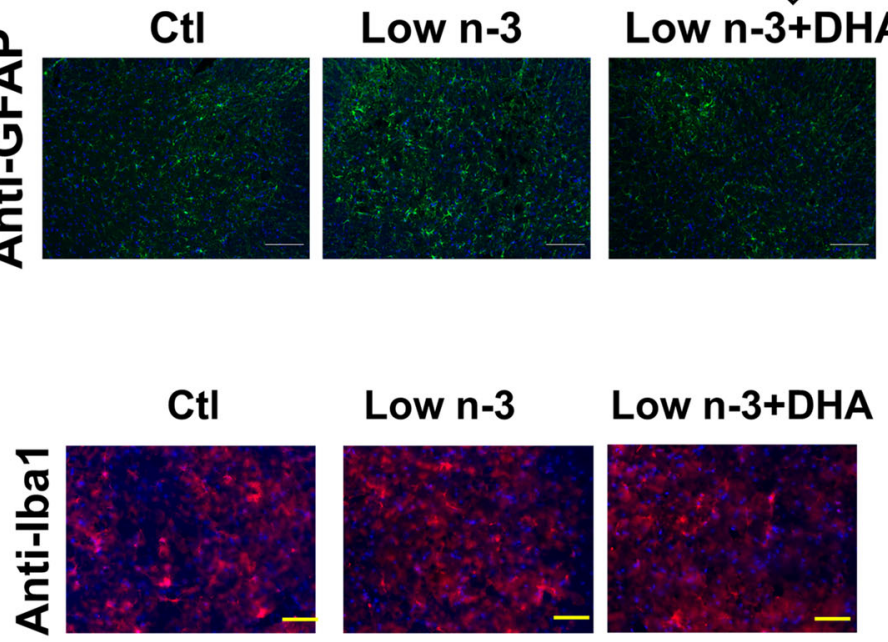

$$
\sum_{\text {Low n-3+DHA }}^{\text {Cll }}
$$
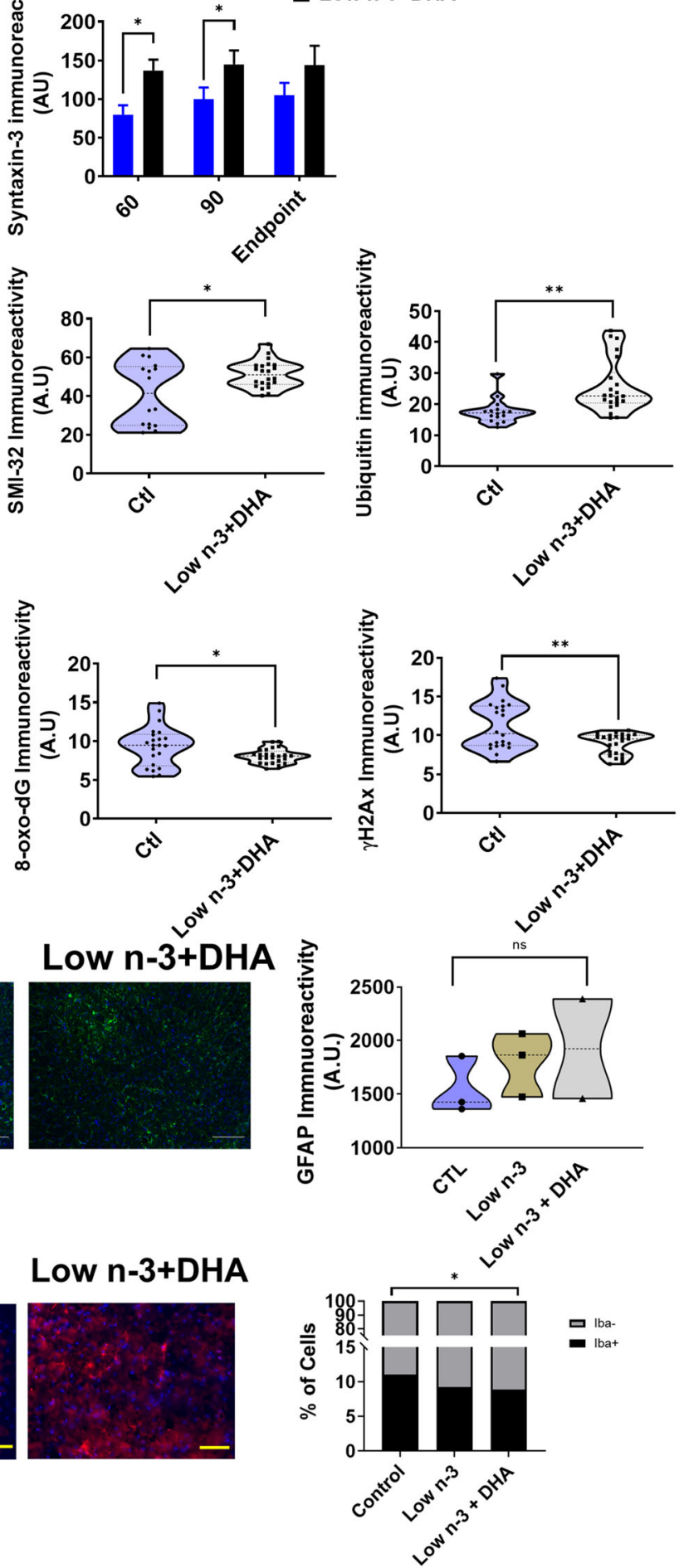

neurodegenerative processes [19], low n-3 levels (without n-3 deficiency) did not lead to relevant changes in survival in comparison with standard linolenic intake, suggesting a crucial and specific role for DHA, compared with other linolenicderived molecules on ALS pathophysiology. Adding complexity to this fact, anti-inflammatory indexes were equally affected in males and females, thereby indicating that males, in addition to changes in fatty acid profiles, are beneficed by yet unknown mechanisms dependent on dietary DHA content.

Protein oxidative damage modifications have been previously linked to Alzheimer's disease [22]. Nevertheless, in the G93A mouse model, protein oxidative damage does not 
Fig. 5 Dietary DHA supplementation leads to changes in synaptic components as well as in DNA and protein repair mechanisms in G93 male mice, without changes in astrogliosis or microglial infiltration. (a) Representative Western blot analyses evaluating levels of syntaxin 3 in LSC. In the right panel, the quantitative analyses of Western blot signals, adjusted to tubulin content. (b) Representative confocal images (left) and quantitative analyses (right) of neurofilament (SMI-32) and ubiquitin immunoreactivities in whole LSC sections of 90-day-old G93A male mice. (c) Representative confocal images (left) and quantitative analyses (right) of DNA repair mechanisms $(\gamma \mathrm{H} 2 \mathrm{Ax})$ and DHA-related decreases in DNA oxidative damage (8-oxo-dG) in whole LSC sections of 90-dayold G93A male mice, measured in immunohistochemistry. (d) Representative confocal images showing a lack of DHA-related changes in GFAP immunostaining in whole SC sections of 90-day-old G93A male mice, quantified in right panels. (e) Representative confocal images showing low-n-3 and low-n-3 + DHA-related decreases in the proportion of Ibal immunoreactive cells in whole SC sections of 90-day-old G93A male mice, quantified in right panels. In (a), bars indicate mean values, whereas error bars represent \pm S.E.M ( $n=4$ experiments). Points in (b), (c), and (d) indicate different sections, belonging to at least 3 different animals for each dietary treatment. Differences between dietary groups were evaluated by Student's $t$ test analyses (or $\chi^{2}$ test for (e)), being ${ }^{*} p<0.05$ and $* * p<0.01$. Scale bars in (b) and (c) are $1000 \mu \mathrm{m}$ long and in (d) and (e) are $100 \mu \mathrm{m}$ and $50 \mu \mathrm{m}$ long, respectively follow a clear tendency during disease progression. Moreover, although dietary n-3 fatty acid intake and aging influences protein oxidative damage, we observed no clear correlation regarding diet or age and protein oxidative stress. These markers are not correlated with a better outcome, and the biological processes associated with diet and age should be further studied to elucidate their impact in ALS pathology.

Neuroinflammation is involved in the progression of neurodegeneration in ALS through microglia activation [37]. In this sense, our data suggest that dietary fatty acids play an important role in modulating inflammation. Most of the nonsteroid antiinflammatory drugs, like aspirin, are cyclooxygenase inhibitors and have been largely studied. Cyclooxygenase activity, using AA as a precursor, promotes inflammation and subsequent tissue damage. Prostaglandins, lipid AA derivatives, are increased in the serum of ALS patients [38]. Furthermore, inhibiting prostaglandin synthesis with anti-inflammatory reduces pathological features, such as loss of motor neurons in the spinal cord, and prolongs survival in G93A mice [35]. DHA has been postulated as an inflammatory-resolution promoter mainly through the action of resolvins, metabolic products of DHA enzymatic oxidation. These molecules are PPAR agonists, a transcription

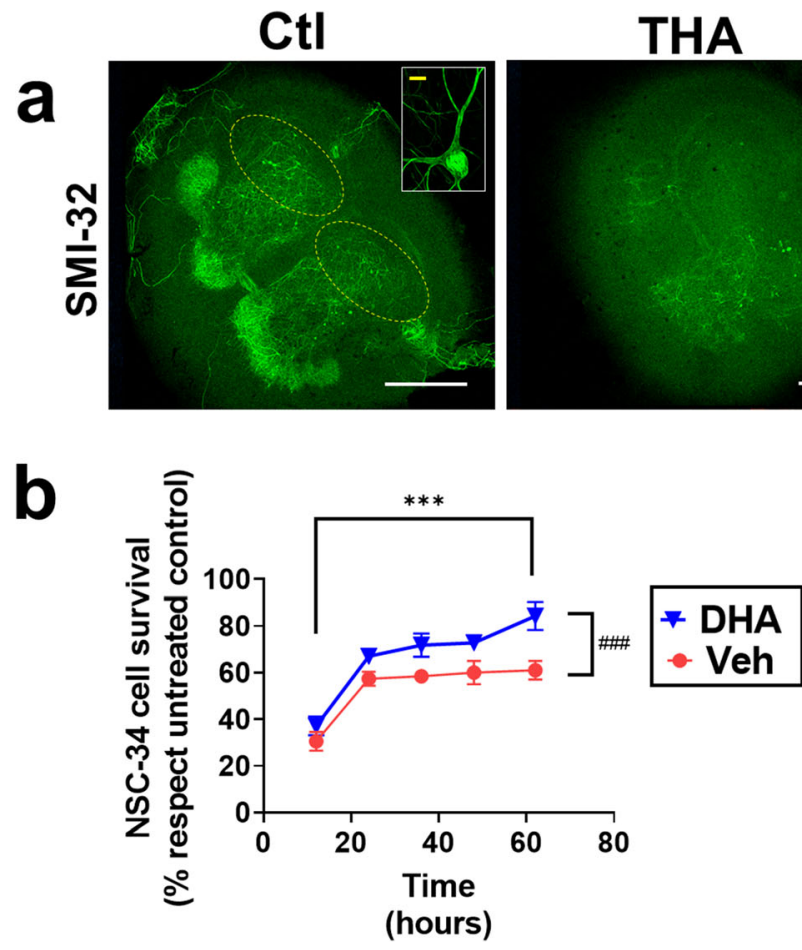

Fig. 6 DHA is neuroprotective in experimental paradigms of motor neuron death. (a) Motor neuron loss induced by THA treatment involves an $\alpha$-tocopherol and DHA-sensitive mechanism. Representative confocal images showing the effect of excitotoxicity in the number of SMI-32-positive cells, compatible with motor neurons (inset showing a magnification) in ventral horns (identified by a yellow ellipse) of rat spinal cord organotypic cultures. Right panel shows the quantitative analyses of these micrographs evidencing diminished motor neuron cell number induced by THA, and this effect being prevented by

DHA and $\alpha$-tocopherol. In (b), DHA $(100 \mu \mathrm{M})$ is able to prevent loss of viability of the motor neuron cell line NSC-34 induced by $\mathrm{H}_{2} \mathrm{O}_{2}$ $(0.1 \mathrm{mM})$. Graphs represent the means \pm S.E.M. ( $n=4$ experiments). In (a), points indicate the number of motor neurons of different slices, belonging to at least 4 different experiments. Numbers in (a) indicate the $p$ value after post hoc analyses in 1-way ANOVA. In (b), $* * * p<0.0001$ with reference to time effect and ${ }^{\# \#} p<0.0001$ with reference to DHA effects by 2-way ANOVA. Scale bars in (a) are $100 \mu \mathrm{m}$ long (white bar) and $10 \mu \mathrm{m}$ long (yellow bar, inset) 
factor which downregulates NF-kB, preventing inflammation and apoptosis [39]. Another anti-inflammatory mechanism of DHA is the competition of cellular incorporation with AA [40], therefore preventing the production of prostaglandins. Our data demonstrate the capacity of dietary DHA to significantly influence cytokine levels in plasma, supporting the potential modulatory effect of dietary DHA in neuroinflammation in this model. Of note, despite we observed a low-n-3-induced decrease in Ibal positivity in the spinal cord, in common with previous data with EPA supplementation [34], this effect was not specific of DHA, as we observed in both low-n-3 diets. Contrasting also with the EPA supplementation experiment, our samples did not show any change of astrogliosis. All in all, these data highlight the complexity of the effects of DHA and other n-3 fatty acids in this ALS model.

We acknowledge, as limitations of the present work, that we did not offer an accurate count of motor neurons in spinal cords from the G93A mice. However, the fact that in 2 separate tissular and cellular models of motor neuron demise, DHA (either alone or in combination with $\alpha$-tocopherol) was able to prevent motor neuron loss, could allow us to hypothesize a similar effect in vivo. We also acknowledge some caveats, such as the increased ubiquitin immunostaining induced by DHA treatments. Usually, ubiquitin is considered a marker of defective proteostasis, but in our case, this might be viewed as a compensatory response.

Concerning potential translation of these results to the human disease context, nutrition for ALS management may be relevant for several reasons. Reports indicate that diet, specifically n-3 fatty acid consumption, plays an important role in ALS progress and may delay the onset of ALS [41]. These data support nutritional intervention as an interesting field to improve the quality of life of ALS patients, but specific issues (such as the interaction with gender) remain open to exploring further venues of dietary personalization in ALS care.

In conclusion, DHA exerts neuroprotective effects in male, but not in female, hSOD1-G93A mouse model of ALS. Further investigations are needed to elucidate this genderdiet interaction. Our data suggest that specific dietary fatty acid composition might be considered in the management of ALS patients.

Acknowledgments We thank D. Argilés and M. Martí for their skillful assistance. Grants were received from the Instituto de Salud Carlos III (PI 17-000134) to MPO and from the Generalitat de Catalunya 2017SGR696 to RP. PT is a predoctoral fellow from the Ministerio de Educacion (FPU16/01446). Support was also received in the form of a FUNDELA Grant, RedELA-Plataforma Investigación and the Fundació Miquel Valls (Jack Van den Hoek donation). FEDER funds are acknowledged ("A way to make Europe").

Compliance with Ethical Standards All experimental procedures were approved by the Institutional Animal Care Committee of the University of Lleida, according to local laws and to the Directive 2010/63/EU of the European Parliament

\section{References}

1. Bruijn LI, Miller TM, Cleveland DW. Unraveling the mechanisms involved in motor neuron degeneration in ALS. Annu. Rev. Neurosci. 2004;27:723-749.

2. Rosen DR, Siddique T, Patterson D, Figlewicz DA, Sapp P, Hentati $\mathrm{A}$, et al. Mutations in $\mathrm{Cu} / \mathrm{Zn}$ superoxide dismutase gene are associated with familial amyotrophic lateral sclerosis. Nature. 1993;362:59-62.

3. Gurney ME, Pu H, Chiu AY, Dal Canto MC, Polchow CY, Alexander DD, et al. Motor neuron degeneration in mice that express a human $\mathrm{Cu}, \mathrm{Zn}$ superoxide dismutase mutation. Science. 1994:264:1772-1775.

4. Blasco H, Guennoc AM, Veyrat-Durebex C. Amyotrophic lateral sclerosis: a hormonal condition? Amyotrophic Lateral Sclerosis 2012;13:585-588.

5. Ajroud-Driss S, Siddique T. Sporadic and hereditary amyotrophic lateral sclerosis (ALS). Biochim. Biophys. Acta. 2015;1852:679684.

6. Singh M. Essential fatty acids, DHA and human brain. Indian J Pediatr. 2005;72:239-242.

7. Serhan CN, Chiang N, Van Dyke TE. Resolving inflammation: dual anti-inflammatory and pro-resolution lipid mediators. Nat. Rev. Immunol. 2008;8:349-361.

8. Jackson PA, Reay JL, Scholey AB, Kennedy DO. DHA-rich oil modulates the cerebral haemodynamic response to cognitive tasks in healthy young adults: a near IR spectroscopy pilot study. Br. J. Nutr. 2012;107:1093-1098.

9. Chytrova G, Ying Z, Gomez-Pinilla F. Exercise contributes to the effects of DHA dietary supplementation by acting on membranerelated synaptic systems. Brain Res. 2010;1341:32-40.

10. Guesnet P, Alessandri J-M. Docosahexaenoic acid (DHA) and the developing central nervous system (CNS) - Implications for dietary recommendations. Biochimie. 2011;93:7-12.

11. Lee LK, Shahar S, Chin A-V, Yusoff NAM. Docosahexaenoic acidconcentrated fish oil supplementation in subjects with mild cognitive impairment (MCI): a 12-month randomised, double-blind, placebo-controlled trial. Psychopharmacology. 2013;225:605-612.

12. Ilieva EV, Ayala V, Jové M, Dalfó E, Cacabelos D, Povedano M, et al. Oxidative and endoplasmic reticulum stress interplay in sporadic amyotrophic lateral sclerosis. Brain. 2007;130:3111-3123.

13. Hernando S, Requejo C, Herran E, Ruiz-Ortega JA, MoreraHerreras T, Lafuente JV, et al. Beneficial effects of n-3 polyunsaturated fatty acids administration in a partial lesion model of Parkinson's disease: The role of glia and NRf2 regulation. Neurobiol. Dis. 2019;121:252-262.

14. Srikanth M, Chandrasaharan K, Zhao X, Chayaburakul K, Ong WY, Herr DR. Metabolism of Docosahexaenoic Acid (DHA) Induces Pyroptosis in BV-2 Microglial Cells. Neuromolecul. Med. 2018;20: 504-514.

15. Zhou M-M, Ding L, Wen M, Che H-X, Huang J-Q, Zhang T-T, et al. Mechanisms of DHA-enriched phospholipids in improving cognitive deficits in aged SAMP8 mice with high-fat diet. J. Nutr. Biochem. 2018;59:64-75.

16. Ajith TA. A Recent Update on the Effects of Omega-3 Fatty Acids in Alzheimer's Disease. Curr Clin Pharmacol. 2018;13:252-260.

17. Silva RV, Oliveira JT, Santos BLR, Dias FC, Martinez AMB, Lima CKF, et al. Long-Chain Omega-3 Fatty Acids Supplementation Accelerates Nerve Regeneration and Prevents Neuropathic Pain Behavior in Mice. Front. Pharmacol. 2017;8:723.

18. Cacabelos D, Ayala V, Ramírez-Nunez O, Granado-Serrano AB, Boada J, Serrano JCE, et al. Dietary lipid unsaturation influences survival and oxidative modifications of an amyotrophic lateral sclerosis model in a gender-specific manner. Neuromolecul. Med. 2014;16:669-685. 
19. Calon F, Lim GP, Yang F, Morihara T, Teter B, Ubeda O, et al. Docosahexaenoic acid protects from dendritic pathology in an Alzheimer's disease mouse model. Neuron. 2004;43:633-645.

20. Muntané G, Janué A, Fernandez N, Odena MA, Oliveira E, Boluda $\mathrm{S}$, et al. Modification of brain lipids but not phenotype in alphasynucleinopathy transgenic mice by long-term dietary n-3 fatty acids. Neurochem. Int. 2010;56:318-328.

21. Bradford MM. A rapid and sensitive method for the quantitation of microgram quantities of protein utilizing the principle of proteindye binding. Anal. Biochem. 1976;72:248-254.

22. Pamplona R, Dalfó E, Ayala V, Bellmunt MJ, Prat J, Ferrer I, et al. Proteins in human brain cortex are modified by oxidation, glycoxidation, and lipoxidation. Effects of Alzheimer disease and identification of lipoxidation targets. J. Biol. Chem. 2005;280: 21522-21530.

23. Calder PC. n-3 polyunsaturated fatty acids, inflammation, and inflammatory diseases. Am. J. Clin. Nutr. 2006;83:1505S-1519S.

24. Rothstein JD, Jin L, Dykes-Hoberg M, Kuncl RW. Chronic inhibition of glutamate uptake produces a model of slow neurotoxicity. Proc. Natl. Acad. Sci. USA. 1993;90:6591-6595.

25. Rasband WS. US National Institutes of Health, Bethesda, Maryland, USA. http://imagej.nih.gov/ij/. 2011;

26. Dyall SC. Long-chain omega-3 fatty acids and the brain: a review of the independent and shared effects of EPA, DPA and DHA, Front. Aging Neurosci. 2015;7:52.

27. Aguirre N, Beal MF, Matson WR, Bogdanov MB. Increased oxidative damage to DNA in an animal model of amyotrophic lateral sclerosis. Free Radic. Res. 2005;39:383-388.

28. Farg MA, Konopka A, Soo KY, Ito D, Atkin JD. The DNA damage response (DDR) is induced by the C9orf72 repeat expansion in amyotrophic lateral sclerosis. Hum. Mol. Genet. 2017;26:28822896.

29. Keller AF, Gravel M, Kriz J. Live imaging of amyotrophic lateral sclerosis pathogenesis: disease onset is characterized by marked induction of GFAP in Schwann cells. Glia. 2009;57:1130-1142.

30. Ito D, Imai Y, Ohsawa K, Nakajima K, Fukuuchi Y, Kohsaka S. Microglia-specific localisation of a novel calcium binding protein, Iba1. Brain Res. Mol. Brain Res. 1998;57:1-9.

31. Sorge RE, Mapplebeck JCS, Rosen S, Beggs S, Taves S, Alexander JK, et al. Different immune cells mediate mechanical pain hypersensitivity in male and female mice. Nat. Neurosci. 2015;18:10811083 .
32. Kitson AP, Stroud CK, Stark KD. Elevated production of docosahexaenoic acid in females: potential molecular mechanisms. Lipids. 2010;45:209-224.

33. Paterniti I, Impellizzeri D, Di Paola R, Esposito E, Gladman S, Yip $\mathrm{P}$, et al. Docosahexaenoic acid attenuates the early inflammatory response following spinal cord injury in mice: in-vivo and in-vitro studies. J. Neuroinflammation. 2014;11:6.

34. Yip PK, Pizzasegola C, Gladman S, Biggio ML, Marino M, Jayasinghe $\mathrm{M}$, et al. The omega-3 fatty acid eicosapentaenoic acid accelerates disease progression in a model of amyotrophic lateral sclerosis. PLoS One. 2013;8:e61626.

35. de Lima IV A, Bastos LFS, Limborço-Filho M, Fiebich BL, de Oliveira ACP. Role of prostaglandins in neuroinflammatory and neurodegenerative diseases. Mediators Inflamm. 2012;2012: 946813.

36. Khairallah RJ, Sparagna GC, Khanna N, O'Shea KM, Hecker PA, Kristian T, et al. Dietary supplementation with docosahexaenoic acid, but not eicosapentaenoic acid, dramatically alters cardiac mitochondrial phospholipid fatty acid composition and prevents permeability transition. Biochim. Biophys. Acta. 2010;1797:15551562.

37. Frakes AE, Ferraiuolo L, Haidet-Phillips AM, Schmelzer L, Braun $\mathrm{L}$, Miranda CJ, et al. Microglia induce motor neuron death via the classical NF- $\mathrm{kB}$ pathway in amyotrophic lateral sclerosis. Neuron. 2014;81:1009-1023.

38. Iłzecka J. Prostaglandin E2 is increased in amyotrophic lateral sclerosis patients. Acta Neurol. Scand. 2003;108:125-129.

39. Serhan CN, Krishnamoorthy S, Recchiuti A, Chiang N. Novel antiinflammatory-pro-resolving mediators and their receptors. Curr. Top. Med. Chem. 2011;11:629-647.

40. Jaudszus A, Gruen M, Watzl B, Ness C, Roth A, Lochner A, et al. Evaluation of suppressive and pro-resolving effects of EPA and DHA in human primary monocytes and T-helper cells. J. Lipid Res. 2013;54:923-935.

41. Fitzgerald KC, O’Reilly ÉJ, Falcone GJ, McCullough ML, Park Y, Kolonel LN, et al. Dietary $\omega-3$ polyunsaturated fatty acid intake and risk for amyotrophic lateral sclerosis. JAMA Neurol. 2014;71: $1102-1110$

Publisher's Note Springer Nature remains neutral with regard to jurisdictional claims in published maps and institutional affiliations. 